\title{
Las revistas de geografía en Colombia: 20 años después de la institucionalización de la disciplina
}

\author{
Israel Cabeza Morales ${ }^{1}$ \\ Universidad Pedagógica y Tecnológica de Colombia \\ israel.cabeza@uptc.edu.co
}

Recepción: 17/05/2012

Evaluación: 04/06/2012

Aceptación: 03/11/2012

Artículo de Reflexión

DOI: http:/ / dx.doi.org/ 10.9757/Rhela.20.08

\section{RESUMEN}

Se hace una revisión de la evolución de las principales publicaciones académicas en el área de geografía en Colombia, teniendo como referente el carácter reciente de la geografía como profesión en el país; se construye un balance de limitantes para la preservación de este tipo de proyectos editoriales, las perspectivas de futuro para estos y para la disciplina en el país. Todo con el objeto de reconocer el rol determinante

The magazines of geography in Colombia: 20 years after the institutionalization of the discipline

\begin{abstract}
It was done a review of the evolution of the main academic publications in the field of geography in Colombia, taking as an up dated referent of the geography as profession in Colombia; there were built a balance of the limits in order to preserve this kind publishing projects, the
\end{abstract}

de las publicaciones seriadas para la consolidación de la disciplina geográfica y apoyo para su enseñanza, a lo cual se vincula el legado de algunas instituciones y personas a través del trabajo en pro de dichas revistas.

Palabras clave: Revista Historia de la Educación Latinoamericana, sociedad de la información, publicaciones seriadas, geografía.

\section{As revistas de geografia na Colômbia: 20 anos depois da institucionalização da disciplina}

\section{RESUMO}

A análise da evolução das principais publicações acadêmicas no campo da geografia, na Colômbia, tendo como referência a natureza recente da geografia como uma profissão no país, a construção de um equilíbrio dos limites para a preservação deste tipo de projeto editores, as perspectivas futuras para si e para

1 Docente Ocasional de la Escuela de Ciencias Sociales -ECS- de la Universidad Pedagógica y Tecnológica de Colombia UPTC-. Integrante del grupo de investigación Vida Territorial Sostenible /Ordenamiento Ambiental del Territorio (VTS/OAT), reconocido por Colciencias (convocatoria, 2012). 
perspectives for the future for the same ones and for the subject in the country. Finally, there were the legacy of some institutions and people to consolidate the knowledge of geography through the magazines.

Key words: History of Latin American Education Journal, society of the information, serial publications, geography, Magazine History of the Latin-American Education. a disciplina no país. Tudo com o objetivo de reconhecer o papel crucial de periódicos para a consolidação da disciplina geográfica e apoio para a sua educação, o que está ligado ao legado de algumas instituições e indivíduos através do trabalho em favor dessas revistas.

Palavras-chave: Revista História da Educação Latino-americana, Sociedade da informação, publicações seriadas, geografia.

\title{
INTRODUCCIÓN
}

\author{
"La palabra eternizada en el papel continua \\ siendo en nuestros días una opción fundamental \\ frente a la desmemoria colectiva -frente al olvido- (...)", \\ Ricardo Gil Otaiza $(2011)^{2}$
}

En octubre del año 2013, un selecto grupo de profesionales de Colombia conmemoró las dos décadas de la existencia formal de la geografía como profesión este país. Por fortuna luego de avatares y trabajo mancomunado, hoy parecen recogerse buenos frutos de una disciplina aún de unos pocos, quienes se han esforzado hasta el punto de que a la fecha la formación posgraduada en esta área del saber llega a 2 programas doctorales y 4 a nivel de maestría; sin duda alguna, esto puede parecer poco para quienes conocen la realidad de la disciplina en países de la región como México y Brasil, pero la solidez consolidada hasta ahora se constituye en un valor agregado para el selecto grupo de los geógrafos colombianos, y es a su vez reconfortante para quienes hasta ahora se aproximan a la disciplina.

La historia del saber geográfico en Colombia, ${ }^{3}$ a través de los procesos de educación formal, puntualmente desde su inserción en la vida universitaria, parece ser anecdótica, pues para 1984, cuando no se contaba con formación universitaria de base en el área, a partir de algunos cuerpos profesorales

2 Ricardo Gil Otaiza, El papel contra el olvido. (Mérida: Universidad de los Andes, Talleres Gráficos ULA, 2011), 323.

3 Con esta expresión se hace alusión a lo propiamente geográfico, pues se deja de lado el precedente de Formación en Ingeniería geográfica, que ofreció desde inicios de la década del setenta la Universidad de Bogotá Jorge Tadeo Lozano y que curiosamente fue cerrado en el año 1993. Ver: José Agustín Blanco Barros. La ciencia geográfica en la historia de Colombia. (Bogotá: Sociedad Geográfica de Colombia-Academia de Ciencias Geográficas, s.f.) www.sogeocol.edu.co/documentos/01laci.pdf (20 de noviembre, 2012) 
de ciencias sociales, principalmente licenciaturas, y de sus profesores recién cualificados en la disciplina fuera de las fronteras nacionales, se propende por las tareas para la gestación de un programa de formación a nivel de maestría para satisfacer las necesidades del país y de los licenciados en Ciencias Sociales que van encargarse de apoyar este tipo de iniciativas, y asumen el reto de formar a las primeras generaciones de geógrafos colombianos. ${ }^{4}$

Esta iniciativa fue precedida por la conformación de grupos y clubes de estudio que posibilitan el surgimiento de la Asociación Colombiana de Geógrafos ACOGE, ${ }^{5}$ en el año 1967, en la sala geográfica Francisco José de Caldas, sitio en el que se desarrollan las reuniones de claustro de los profesores de la Escuela de Ciencias Sociales de la Universidad Pedagógica y Tecnológica de Colombia (UPTC). Es a partir de esta iniciativa que se empiezan a formar las primeras generaciones de geógrafos colombianos, quienes principalmente provenían de la formación en ciencias sociales.

Así, luego de varios años y del surgimiento de programas de pregrado en geografía, surge la Ley 78 de 1993, ${ }^{6}$ también conocida como ley del geógrafo, por la cual se reglamenta el ejercicio de la profesión de Geógrafo y se dictan otras disposiciones, entre las cuales se contempla la creación del Colegio Profesional de Geógrafos, entidad encargada de expedir las tarjetas profesionales en el área; paralelo a esto pareciera oficializarse la expansión de la disciplina con la gestación de los primeros programas de formación universitaria a nivel de pregrado, entre ellos los de las universidades del Cauca, Nariño, Córdoba, y por supuesto la Universidad Nacional de Colombia.

Sin duda alguna, en este proceso de consolidación por el cual aún hoy se trabaja, las publicaciones seriadas han desempeñado un rol de apoyo significativo a los intentos por la solidez constitutiva de una comunidad académica de geógrafos en el país, especialmente a través de su contribución como soporte a los procesos de enseñanza-aprendizaje de la geografía.

Pese a que las publicaciones extranjeras (en idiomas distintos al español) fueron determinantes para la actualización de los primeros geográfos del

4 Así nace el Programa de Estudios de Posgrado en Geografía (Convenio entre la Universidad Pedagógica y Tecnológica de Colombia UPTC y el Instituto Geográfico Agustín Codazzi, IGAC), que desde el año 2010 ofrece formación a nivel doctoral.

5 Ver: Gustavo Montañez Gómez, "Elementos de historiografía de la Geografía colombiana" Revista Estudios Sociales No. 3(1999): 9-28.

6 Reglamentada por Colombia, Congreso de la República, Decreto № 1801 de 1995. 
país ${ }^{7}$ y aún tienen gran relevancia por las actuales dinámicas de producción de conocimiento científico, las publicaciones seriadas nacionales se constituyen en un hito para la difusión del conocimiento geográfico entre los adeptos que paulatinamente se vinculaban a la disciplina, por ello es pertinente hacer un recorrido histórico para la reconstrucción de este proceso, para responder a la pregunta de ¿cuál ha sido su aporte a la disciplina en Colombia?; pues si bien, en la actualidad ha crecido el número de programas de pregrado y posgrado en geografía, las publicaciones académicas seriadas específicas en el área siguen siendo un referente significativo.

El presente artículo es producto de la revisión a las características de las distintas publicaciones en el área de geografía y el análisis de las dinámicas que han circundado cada uno de estos proyectos editoriales, con el fin de asociar la evolución de las publicaciones con el posicionamiento de la disciplina en el territorio nacional.

\section{Las publicaciones seriadas en geografía, una preocupación de la UPTC}

Uno de los principales referentes para las publicaciones en geografía en la UPTC lo constituye el Club Geográfico, que surge en la especialidad en ciencias sociales, posteriormente convertida en licenciatura. Además de impulsar la organización de otros clubes de estudio, intentó posicionar su quehacer con una publicación denominada Huellas geográficas, de la que solo se conoce un ejemplar publicado en diciembre 1981, con el número 1 (imagen 1), esta publicación se destaca por la dirección editorial de la profesora Clara Mateus Cárdenas y la asesoría, a través del Club Geográfico, con el doctor Héctor Fabio Rucinque.

7 Ovidio Delgado Mahecha, Debates sobre el espacio en la geografia contemporánea. (Bogotá: Universidad Nacional de Colombia-Unibiblos, 2003), 9. 


\section{Imagen 1. Portada revista Huellas geográficas}

Fuente: elaboración propia, 2013.

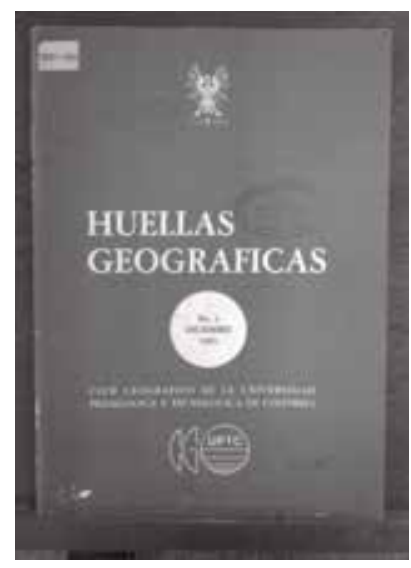

Como bien se cita en la editorial de su primer ejemplar, denominada mensaje inaugural, la Profesora Clara R. Mateus, el Club Geográfico “Fundado por el Doctor Héctor Fabio Rucinque en 1965, antecedió en dos años a la Asociación Colombiana de Geógrafos, y es la primera organización geográfica en el campo universitario colombiano de que se tenga noticia" ${ }^{8}$.

En 1968 surge el Correo Geográfico como publicación de la Asociación Colombiana de Geógrafos (ACOGE) con el patrocinio editorial de la UPTC y bajo la coordinación editorial de Héctor Fabio Rucinque y posteriormente de Carlos Cuervo Escobar, esta publicación, un poco más rica en contenido que Huellas Geográficas, desaparece igual que su antecesora. En el año 1980, bajo el sello editorial de la UPTC surge Trimestre Geográfico ${ }^{9}$, como su nombre lo dice, es una publicación trimestral, la cual fue impulsada por quienes constituían ACOGE, a pesar del empeño esta publicación tuvo que cambiar su periodicidad a anual, sin afectar su nombre, lo que le permitió mantenerse como referente para la disciplina hasta el año 1999, en el cual deja de ser puesta en circulación; entre las personas que impulsaron esta publicación, mediante la tarea de editores se rescatan, además del profesor Rucinque, Ovidio Toro Segura, Nohora Avella y Rodolfo Espinoza.

8 Clara Mateus Cárdenas, "Mensaje inaugural”, Huellas geográficas. No. 1 (1981): 2.

9 El último número de Trimestre Geográfico, correspondiente a 1999 no fue impreso en la UPTC, fue impreso bajo el sello editorial de la Universidad del Valle. 


\section{Imagen 2. Publicaciones geográficas no institucionales}

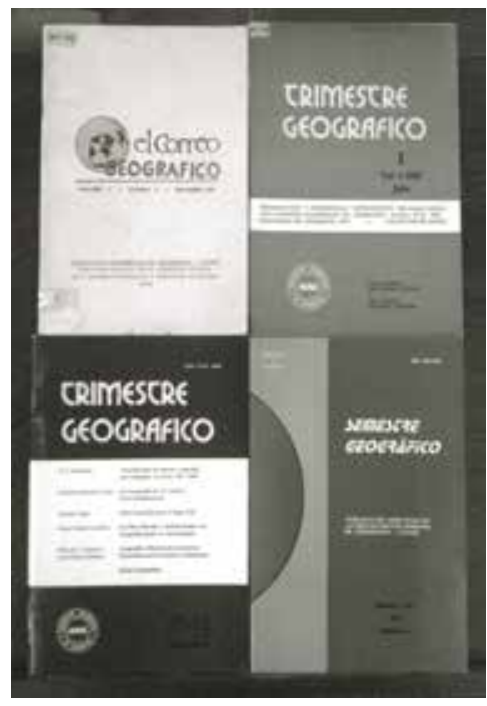

Fuente: elaboración propia, 2013.

La imagen 2 es una muestra de los primeros esfuerzos por la institucionalización de la disciplina, y el intervalo de tiempo en la existencia de dichas publicaciones es evidencia de los inconvenientes y esfuerzos a los que se enfrentaron sus impulsores, entre las publicaciones relacionadas (imagen 2), se evidencia que Trimestre Geográfico fue la publicación de mayor continuidad que ha impulsado ACOGE; finalmente en la imagen también se encuentra Semestre Geográfico, publicación con la que en el 2001 esta asociación hace un esfuerzo para no dejar de lado la tarea editorial y el legado de Trimestre Geográfico, surgiendo así Semestre Geográfico, como publicación arbitrada y también patrocinada bajo el sello editorial de la UPTC; en este mismo año salen los números 1 y 2, correspondientes a cada uno de los semestres de dicho año, pero su continuidad no fue loable.

En 1990, con la pretensión de dar impulso a la disciplina desde el quehacer universitario, dados los intentos poco constantes hasta entonces, surge Apuntes de Geografía (imagen 3), publicación de la Facultad Seccional Chiquinquirá y denominada como revista de divulgación científica, a diferencia de sus anteriores esta va a contar en su número 2 con ISSN y articulándose un poco con la proyección de desarrollo del Departamento de Geografía de la Facultad Seccional Chiquinquirá, se muestra una publicación vinculada a una visión geográfica más contemporánea, articulada al análisis filosófico 
y de las ciencias sociales, sin dejar de lado las temáticas tradicionales de la disciplina, asociadas a la geografía física; la dirección de esta publicación estuvo a cargo del profesor Alberto González Molina, director de dicho departamento, en la UPTC del municipio de Chiquinquirá.

\section{Imagen 3. Revista Apuntes de Geografía}

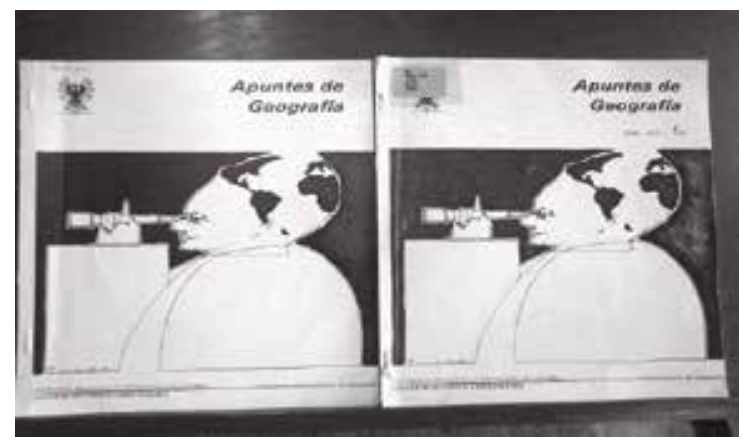

Fuente: elaboración propia, 2013.

Continuando con las iniciativas, y debido a la poca continuidad que logran las anteriores, en mayo de 1991 empieza la puesta en circulación del volumen 1, número 1 de una publicación denominada EPG-GEOGRAFÍA (imagen 4), como herramienta para la difusión de las investigaciones al interior del Programa de Estudios de Posgrado en Geografía EPG (Convenio UPTC-IGAC); la iniciativa estuvo coordinada a través de su Asociación de Exalumnos, entre quienes se destacan Ovidio Delgado Mahecha e Hilda María Sarmiento Gómez, quien coordinó la tarea editorial; de esta publicación que luego de su primer número, combinó los números 3 y 4 en el volumen del año siguiente, se desconoce continuidad.

\section{Imagen 4. EPG- GEOGRAFÍA}

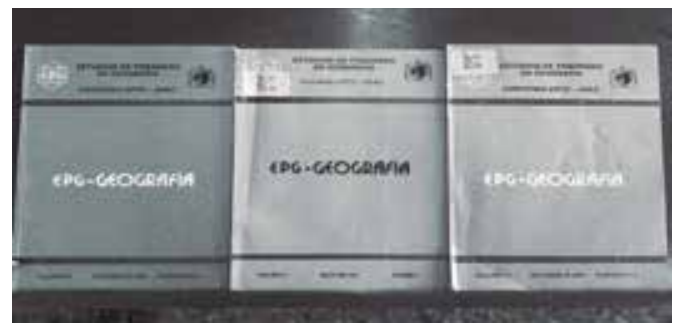


En 1997, nuevamente desde el EPG, aparece una iniciativa aún latente, es Perspectiva Geográfica, definida en sus inicios como la revista de dicho programa de estudios, bajo la coordinación editorial de Ángel Massiris Cabeza; la publicación se va enriqueciendo a lo largo del tiempo con aportes de los docentes invitados para seminarios del EPG y por ellos en años siguientes se hace semestral, pero entre 1999 y 2001 se realiza una impresión anual, en la que se especifica la que cada ejemplar corresponde al primer y segundo semestre del año en que se publica.

Durante el año 2002 se retoma la semestralidad hasta el 2003, cuando a amanera de número especial, por la celebración de los 20 años del EPG, se publica el número 10 como correspondiente al segundo semestre 2003 y primer y segundo semestre 2004. Este número deja ver una Revista más abierta, pensada hacia al mundo, a través de la inclusión de un artículo inicial en inglés, que fue: Geography for the Third Millenium: Inventory and prospect, de la autoría de Anne Buttimer. ${ }^{10}$ A partir del número 11 correspondiente al año 2005 se deja de hablar de semestralidad y se suspende la publicación por dos años hasta 2007, cuando sale el número 12 y retoma una periodicidad anual.

Desde el año 2009 se cambia el número por volumen, para continuar resaltando la secuencia de la publicación y en ese mismo año se pone en circulación el volumen 14; gracias al esfuerzo de algunos y al relevo generacional, así como al crecimiento del EPG, a partir de la creación del programa de Doctorado en Geografía, Perspectiva Geográfica se transforma a partir del volumen 15 (imagen 5), articulándose a la dinámica científica del momento y, por supuesto, ya no como el órgano de difusión de una unidad académica, sino como un referente válido para la proyección del quehacer geográfico en la región. ${ }^{11}$

10 Expresidenta reciente (para la fecha de publicación de la Revista) de la Unión Geográfica Internacional UGI. Integrante del Departamento de Geografia del University College Dublin (Irlanda).

11 Para la fecha de redacción este artículo se informa que en aras de mejor posicionamiento y el aumento significativo en la recepción de artículos, Perspectiva Geográfica volverá a ser una publicación semestral para el año 2014. 


\section{Imagen 5. Evolución de Perspectiva Geográfica}

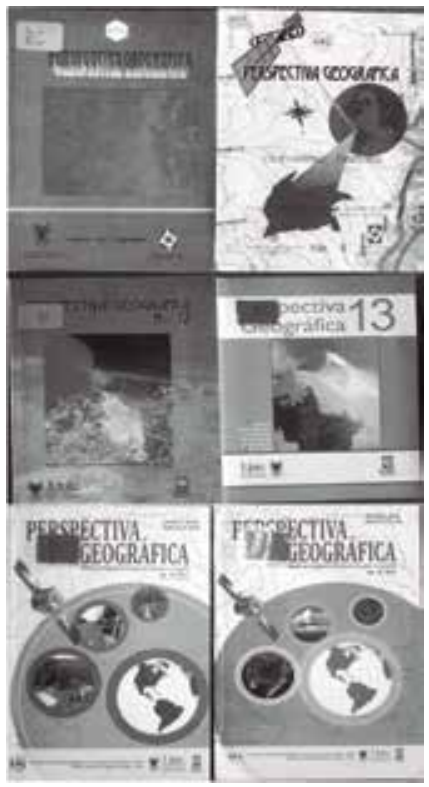

Fuente: elaboración propia, 2013.

\section{De la Universidad Nacional de Colombia y una publicación seriada de gran importancia en geografía}

En el año 1989 surge Cuadernos de Geografía como revista del naciente departamento de geografía de la Universidad Nacional de Colombia, con el apoyo del Instituto Colombiano para el Fomento de la Educación Superior (ICFES) y su Red de Formación Ambiental; para difundir y soportar la actividad de dicha unidad académica, la publicación se mantuvo con el notorio esfuerzo con que suelen subsistir las publicaciones académicas serias en el país, particularmente en disciplinas de reconocido rigor; entre quienes trabajaron en la tarea editorial se destacan, en los roles de director y editor: Ovidio Delgado Mahecha, Joaquín Molano, Antonio Flórez, Luis Carlos Jiménez Reyes, Astrid Ulloa y, hasta la actualidad, John Williams Montoya .

Esta publicación inicialmente semestral, se transforma en 1993 cuando retira de su portada el apoyo del ICFES, reorientando la selección de sus escritos, diseño de portada y declarando periodicidad anual, lo cual le brinda el soporte que permite, en el año 2001, que su comité editorial le otorgue la connotación de Revista Colombiana de Geografía y a partir de ello se insertan 
mejoras, estrategias de visibilización y enriquecimiento de contenidos; por ello a partir del año 2011 la publicación convierte su periodicidad a semestral, lo que ha permitido que a la fecha se mantenga como una publicación de gran reconocimiento a nivel nacional y regional.

\section{Imagen 6. Evolución Cuadernos de Geografía}

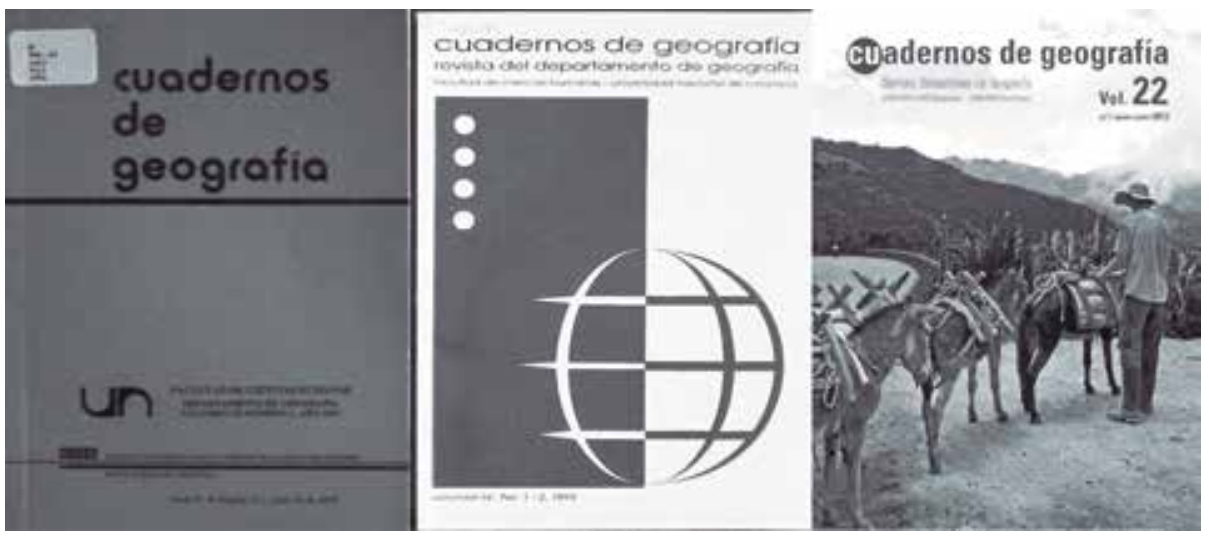

Fuente: elaboración propia, 2013.

La imagen 6 deja ver los cambios significativos que ha tenido la publicación en momentos cruciales para la proyección del saber geográfico y su adaptabilidad a las circunstancias de cada momento, por lo que hoy le es posible disfrutar de reconocimiento.

\section{Otras publicaciones seriadas en geografía}

En el año 2003, por iniciativa de Hector Fabio Rucinque, quien la coordinara editorialmente, se da a conocer la revista GeoTrópico, ${ }^{12}$ como publicación semestral, de carácter digital, impulsada a través un proyecto gestado en la Universidad de Córdoba (Montería, Colombia), denominado Grupo Geo Lat, el cual se constituye en un canal para la interacción de los geógrafos latinoamericanos; sin duda esta iniciativa se constituye en una forma distinta por incentivar la difusión del quehacer de los geógrafos, por ser promotora del libre acceso, pero a pesar de ello no ha logrado cumplir a cabalidad con la periodicidad declarada y a la fecha solo se conocen los volúmenes correspondientes a los años 2003, 2004, 2005, 2010, 2011.

12 Revista Geográfica Internacional electrónica, publicada por el Grupo GEOLAT - Bogotá, Colombia http://www.geotropico.org/ GeoTropTitular.html 
Otra publicación seriada es Entorno Geográfico, impulsada por el Departamento de Geografía de la Universidad del Valle, que cuenta con docentes egresados el Convenio UPTC-IGAC, esta Revista tampoco se encuentra al día en su circulación, a la fecha se prepara el numero 9 correspondiente al año 2011 y se sabe de ella a través de un sitio web ${ }^{13}$ en el que no se encuentran disponibles todos los números publicados. Se desataca la labor editorial de los profesores Jaime Vásquez y Rodolfo Espinoza.

\section{Algunas consideraciones}

Académicos neófitos en el área y próximos a ella suelen estigmatizar la producción en geografía, recopilada por muchas de las publicaciones descontinuadas, como algo de poco valor, directamente relacionado con una visión geográfica reducida, pero se desconoce que la pretensión de quienes escribían y quienes editaban era la de dejar insumos para la pervivencia de la disciplina y, en cierta medida, garantizar mínimos para quienes enseñaban en el área, o deseaban aproximarse a esta. Las publicaciones seriadas en geografía han sido testigos y, a la vez prueba, de la evolución de la disciplina, y constituyen un referente válido, para "el paso de una geografía parroquial a la consolidación de una geografía académica y profesional", ${ }^{14}$ como bien se ha dicho en relación a la evolución de la disciplina, de los ires y venires, en términos de cientificidad, por los que se ha discutido en la geografía colombiana ${ }^{15}$ quizá ello explique la particularidad de algunos de los proyectos editoriales que han existido y su filiación a determinadas corrientes científicas.

Si bien se ha constatado la persistencia por la gestación de una cultura académica a través de las publicaciones en geografía, es de reconocer la existencia de numerosos esfuerzos poco fructíferos, aunque se destaquen las iniciativas de la Universidad Nacional de Colombia y la UPTC a través del (Convenio UPTC-IGAC), lo que ha posibilitado la permanencia y el posicionamiento de sus publicaciones en el área, como lo son Cuadernos de Geografía y Perspectiva Geográfica, hoy publicaciones indexadas a nivel nacional e internacional en el área, con existencia digital.

13 Universidad del Valle, Departamento de Geografía. Revista Entorno Geográfico. http://entornogeográfico.univalle.edu.co/

14 Jhon Williams Montoya Garay, "Geografía contemporánea y geografía escolar: algunas ideas para una agenda en Colombia", Cuadernos de Geografia Vol.: XII No. 1-2 (2003): 6.

15 Héctor Fabio Rucinque, La geografía de entonces y la de ahora... Rápida mirada al desarrollo de la geografia académica en Colombia. (Geografía en Español, Serie GE-Textos Originales [Colombia], No 1). http://www.geografiaenespanol.net/HFR GEtxts.or_1.pdf (29 de noviembre, 2012). 
La tarea editorial hoy, más allá de la difusión de la productividad científica, constituye un elemento clave para el quehacer universitario y para la construcción de líneas de trabajo que permitan la proyección de un área del saber, particularmente sus contribuciones al tratamiento de los problemas que aquejan a la sociedad; de allí que estas dos publicaciones a las que antes se ha hecho mención, hoy sigan trabajando por su visibilización a escalas mayores a los claustros universitarios.

La relación entre la oferta de programas de formación y el número de publicaciones seriadas en el área de geografía es un referente válido para hacer balances e incentivar la producción en el área, pero no es un pretexto para la generación de nuevas publicaciones cuya periodicidad sea cuestionable; la mejor estrategia para la construcción de conocimiento, la consolidación y proyección de un área del saber es el trabajo en red o colaborativo, que para el caso de la geografía plantea la necesidad de que se contemple la posibilidad de proyectos editoriales conjuntos, que alcancen mayor eco y permanencia que iniciativas dispersas. A esto cabe aclarar con gran pertenencia la cita del Profesor Rucinque:

Si bien la vinculación gremial puede ser interesante y necesaria para la cohesión de nuestra comunidad alrededor de algunos propósitos comunes, puede ser prescindible. Lo que síes mandatorio e irrenunciable es la obligación individual para quien escogió esta como su carrera de vida, labrar poco a poco su formación con la excelencia profesional y científica como su meta personal. ${ }^{16}$

Las contundentes palabras de la cita anterior invitan a seguir construyendo a cualquier precio, mientras no se pierda la esencia, entendida como la preocupación por el trabajo en pro de la disciplina, que hagan de la geografía ciencia y profesión con reconocimiento nacional de eco constante.

\section{CONCLUSIÓN}

Ha sido evidente la pretensión de los cuerpos no colegiados, como las asociaciones académicas, los clubes para la consolidación de la geografía, a través de las publicaciones seriadas que han intentado mantenerse en

16 Héctor Fabio Rucinque, La geografia de entonces y la de ahora... Rápida mirada al desarrollo de la geografia académica en Colombia. (Geografía en Español, Serie GE-Textos Originales [Colombia], № 1). http://www.geografíaenespanol. net/HFR GEtxts.or_1.pdf (29 de noviembre, 2012). 
el área. Es de reconocer la preponderancia que ha tenido la UPTC para la consolidación de la geografía como área del saber en la formalidad de la educación superior del país; así como el peso que han tenido las ciencias sociales y consigo los licenciados en este campo, pues han sido los impulsores de la formación de las actuales generaciones de geógrafos que siguen trabajando por la visibilización de la disciplina.

Igualmente, la constancia de quienes en una y / o varias ocasiones han asumido la tarea editorial con el peso de incentivar a la producción investigativa, a pesar de las múltiples limitantes, seguirá siendo el motor para el desarrollo de la disciplina, muchos de estos gestores de cultura académica para el caso de las publicaciones en geografía son grandes maestros entre quienes no sobra destacar a: Héctor Rucinque, Gustavo Montañez, Ovidio Delgado, Hilda María Sarmiento y Ángel Massiris.

La necesidad de contemplar el esfuerzo de las dos publicaciones que, dada la historia reciente de la disciplina, aún se mantienen con rigor y proyección como pilares para el desarrollo de la investigación y su divulgación, a las cuales ojalá la comunidad geográfica nacional no deje de tener presente, hace que gran parte del colectivo geográfico nacional (integrante o no de una asociación $\mathrm{u}$ alguna sociedad particular) reconozca la pertinencia de estas dos publicaciones: Perspectiva Geográfica y Cuadernos de Geografía.

Sin las ilusiones, el esfuerzo y el reconocimiento de las publicaciones que han existido en la historia de la geografía como disciplina formal en Colombia, la cita con la que se encabeza este artículo sería una gran falacia.

\section{FUENTES}

Apuntes de Geografía, Tunja, Universidad Pedagógica y Tecnológica de Colombia, Números: 1 y 2.

Decreto $N^{\circ} 1801$ de 1995, Ministerio de Educación Nacional. Diario Oficial No.42.059, de 23 de octubre de 1995.

El Correo Geográfico, Tunja, Universidad Pedagógica y Tecnológica de Colombia, Número: 1. EPG-Geografía, Tunja, Universidad Pedagógica y Tecnológica de Colombia, Números: 1 al 4. Huellas Geográficas, Tunja, Club geográfico de la Universidad Pedagógica y Tecnológica de Colombia, Número 1. 
Ley 78 de 1993, Congreso de la República de Colombia. Diario Oficial No.41.081, de 19 de octubre de 1993.

Revista Cuadernos de Geografía, Bogotá, Universidad Nacional de Colombia, Volúmenes: 1 al 22.

Revista Perspectiva Geográfica, Tunja, Universidad Pedagógica y Tecnológica de Colombia, Volúmenes: 1 al 16.

Semestre Geográfico, Tunja, Universidad Pedagógica y Tecnológica de Colombia, Números: 1 y 2.

Trimestre Geográfico, Tunja, Universidad Pedagógica y Tecnológica de Colombia, Números: 1 al 19.

\section{REFERENCIAS}

Blanco Barros, José Agustín, La ciencia geográfica en la historia de Colombia. Bogotá: Sociedad Geográfica de Colombia-Academia de Ciencias Geográficas, (s.f.). www.sogeocol.edu.co/ documentos / 01laci.pdf (Marzo de 2012)

Delgado Mahecha, Ovidio. Debates sobre el espacio en la geografía contemporánea. Bogotá: Universidad Nacional de Colombia-Unibiblos, 2003.

Gil Otaiza, Ricardo. El papel contra el olvido. Mérida (Venezuela): Universidad de los Andes, Talleres Gráficos ULA, 2011.

Mateus Cárdenas, Clara. “Mensaje Inaugural”, Huellas Geográficas. No.. 1 (1981): 2-3.

Montañez, Gustavo. "Elementos de historiografía de la Geografía colombiana”. Revista Estudios Sociales, No. 3 (1999): 9-28.

Montoya, Jhon. “Geografía contemporánea y geografía escolar: algunas ideas para una agenda en Colombia". Cuadernos de Geografía, Vol. XII No. (1-2), (2003): 3-27.

Rucinque, Héctor, La geografía de entonces y la de ahora... Rápida mirada al desarrollo de la geografía académica en Colombia. Geografía en Español, Serie GE-Textos Originales, No. 1, 2011. http: / / www.geografíaenespanol. net/HFR_GEtxts.or_1.pdf (Marzo de 2012)

Universidad del Valle, Departamento de Geografía. Revista Entorno Geográfico. http:// entornogeográfico.univalle.edu.co/

Cabeza Morales, Israel. "Las revistas de geografía en Colombia: 20 años después de la institucionalización de la disciplina". Revista Historia de la Educación Latinoamericana. Vol. 15 No, 20, (2013): 175 - 188. 